\title{
Hemorragia intracraniana secundária à derivação ventricular
}

\author{
Carlos Umberto Pereira*, Antonio Ribas ${ }^{\star \star}$, João Domingos Barbosa Carneiro Leão***, \\ Alvino Dutra da Silva***, Priscila Cardoso Braz ${ }^{* \star \star *}$ \\ Serviço de Neurocirurgia do Hospital João Alves Filho (Aracaju, SE) e Serviço de Neurocirurgia da Clínica Santa Lücia (Rio de \\ Janeiro, RJ)
}

\section{RESUMO}

Complicações decorrentes do tratamento cirúrgico de hidrocefalia são bastante freqüentes. O risco do desenvolvimento de hemorragia intracraniana após inserção ou retirada do cateter ventricular tem sido considerado raro. No entanto, pode desencadear hemorragia intracraniana (extradural, subdural, intracerebral e intraventricular).

Os autores, num estudo compreendido no periodo de julho de 1992 a novembro de 2002, identificaram 20 casos de hemorragia intracraniana em decorrência do manuseio do cateter ventricular. Foram observados: nove pacientes com hematoma subdural, quatro com hematoma extradural, quatro com hematoma intraparenquimatoso, dois com hemorragia intraventricular e um com sangramento intratumoral. A idade dos pacientes variou de 6 meses a 28 anos. Dez destes necessitaram de tratamento cirúrgico do hematoma. Óbito ocorreu em quatro dos operados.

São discutidas as medidas para minimizar as complicações hemorrágicas após instalação da derivação.

\section{PALAVRAS-CHAVE}

Hemorragia intracraniana. Hidrocefalia. Complicação de derivação ventriculoperitoneal.

\begin{abstract}
Intracranial hemorrhage due to ventricular shunting

Intracranial hematoma as a complication of shunt procedures for treatment of hydrocephalus is very rare.

The authors report a series of 20 cases of intracranial hemorrhage associated to shunting procedures, observed from July 1992 to November 2002. The age of the patients raged from 6 months to 28 years. There were: nine patients with subdural hematoma, four with epidural hematoma, four with intracerebral hematoma, two with intraventricular haemorrhage and one case of intratumoral bleeding. Ten patients needed surgical removal of the hematoma. Death occurred in four.

The measures directed to avoid this complication are discussed.
\end{abstract}

\section{KEYWORDS}

Intracranial hemorrhage. Hydrocephalus. Shunt complications.

\section{Introdução}

Hemorragia intracraniana pode ocorrer durante um procedimento neurocirúrgico considerado simples, como a inserção do cateter ventricular para o tratamento de hidrocefalia, e, ocasionalmente, apresenta sérias complicações neurológicas.

Atualmente, a tomografia computadorizada (TC) e a ressonância magnética (RM) são feitas rotineiramente no pós-operatório do tratamento da hidrocefalia, para identificação da localização do cateter e avaliação da redução do sistema ventricular. A identificação de complicações hemorrágicas intracranianas sem esses recursos era rara, mas ocasionalmente detectada por mau funcionamento do sistema de derivação ou por meio de uma lesão expansiva.

As hemorragias intracranianas ocasionadas pela inserção do cateter ventricular ocorrem no espaço

\footnotetext{
*Professor Adjunto Doutor do Departamento de Medicina da Universidade Federal de Sergipe e Neurocinurgião do Hospital João Alves Filho. Aracaju, SE.

**Neurocirurgião do Departamento de Ncurocirurgia da Clínica Santa Lúcia. Rio de Janeiro, RJ.

***Neurocirurgião do Hospital João Alves Filho. Aracaju, SE.

****Doutoranda de Medicina da Universidade Federal de Sergipe. Aracaju, SE.
} 
extradural, subdural, intracerebral e intraventricular. Vários fatores têm sido relacionados com essa complicação: tentativas múltiplas de punção do ventrículo, perfuração do plexo coróide, colocação imprópria do cateter dentro do parênquima cerebral e lesão de vaso cerebral ${ }^{7,36}$. Quando essas hemorragias são pequenas, geralmente são assintomáticas e cursam com bom prognóstico.

Os autores apresentam sua experiência em 20 pacientes que tiveram hemorragia intracraniana como complicação na utilização do cateter ventricular. Discutem as possíveis causas, tratamento e prognóstico.

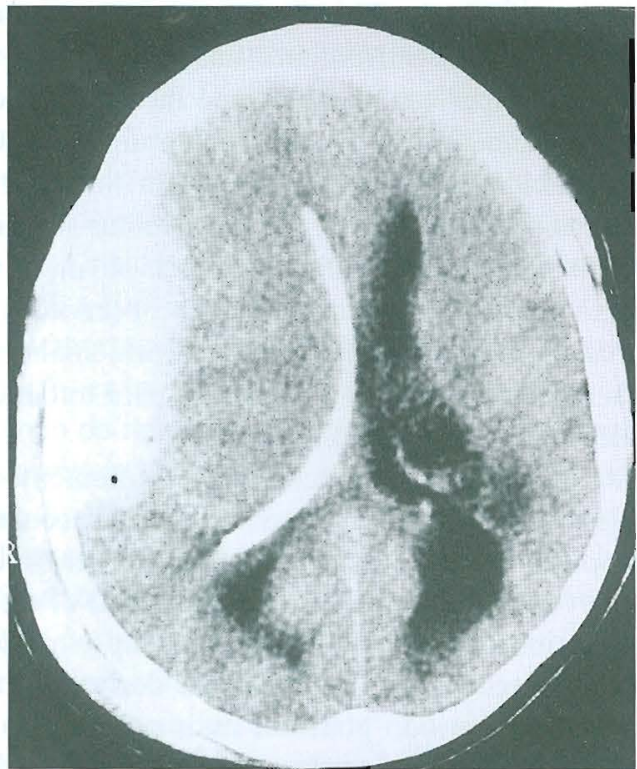

Figura 1 - TC: hematoma extradural parietal direito e presença de cateter de derivação.

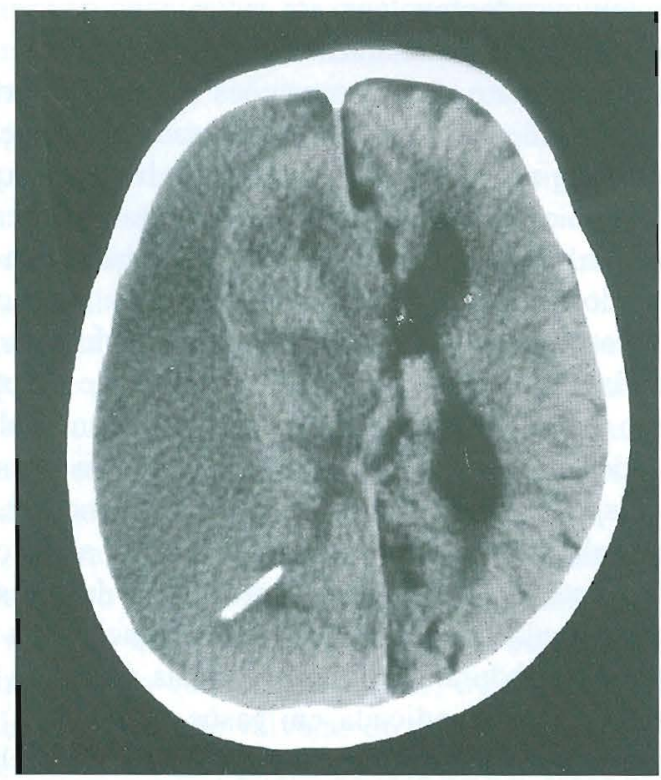

Figura 2 - TC: hematoma subdural frontoparietal direito $e$ presença do cateter.

\section{Casuistica}

Foram analisados 20 pacientes que apresentaram hemorragia intracraniana como complicação no emprego de cateter ventricular, nos Serviços de Neurocirurgia do Hospital João Alves Filho (Aracaju, SE) e Serviço de Neurocirurgia da Clínica Santa Lúcia (Rio de Janeiro, RJ), ocorrida no período de julho de 1992 a novembro de 2002. A idade dos pacientes variou de 6 meses a 28 anos. A localização da hemorragia foi: subdural em nove casos, extradural em quatro, intracerebral em quatro, intraventricular em dois e intratumoral em um (Figuras 1 a 5).

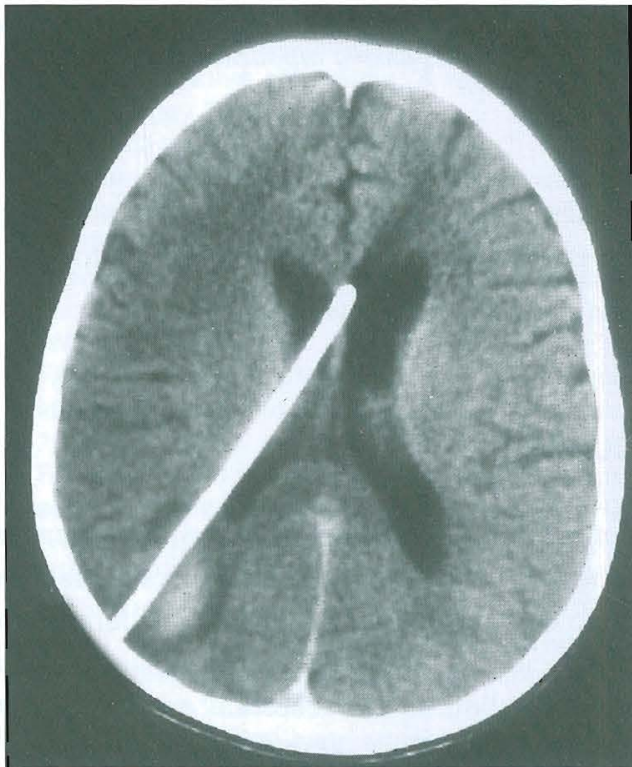

Figura 3-TC: hematoma intracerebral no trajeto do cateter ventricular.

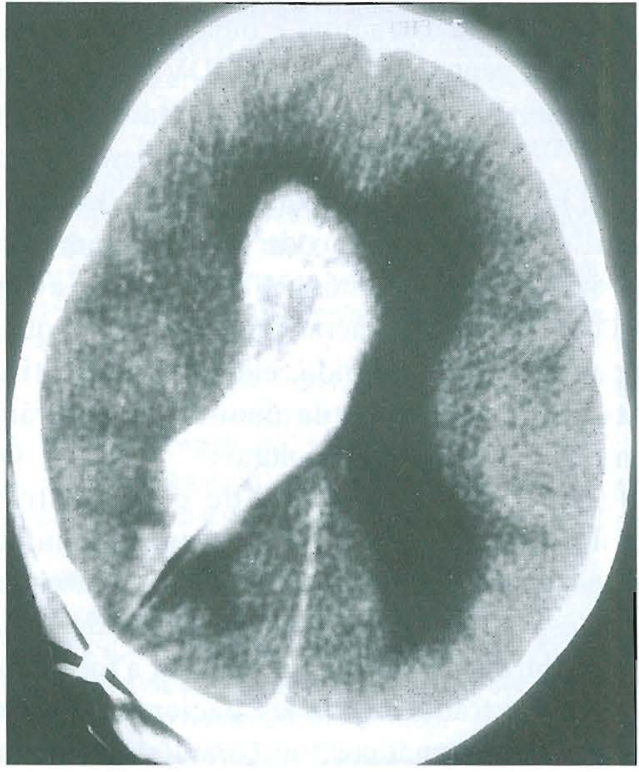

Figura 4 - TC: hemorragia no ventrículo lateral direito, onde foi introduzido o cateter. 


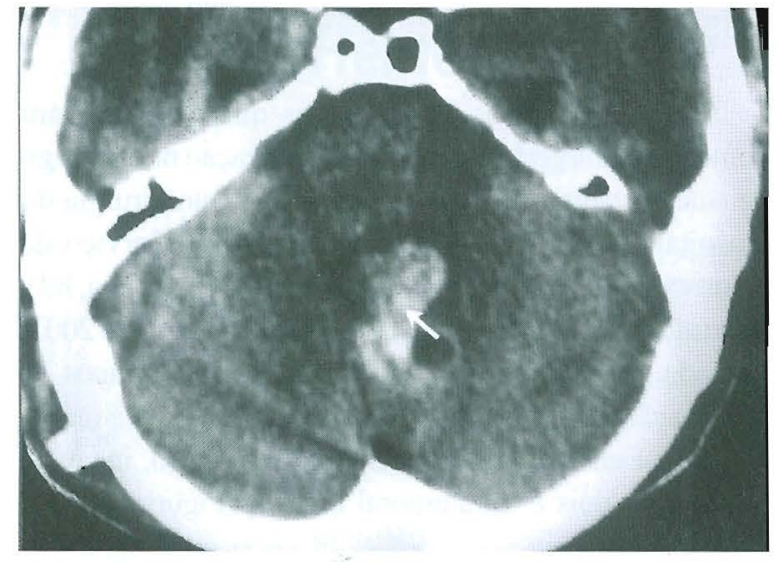

Figura 5 - TC: hemorragia no quarto ventrículo e uma lesão expansiva hipodensa (seta).

Dez pacientes foram submetidos a tratamento cirúrgico do hematoma e quatro evoluíram para óbito.

\section{Discussão}

Hemorragia intracraniana como complicação da introdução do cateter ventricular para o tratamento cirúrgico de hidrocefalia tem sido considerada evento raro. A hemorragia pode localizar-se no espaço extradural, subdural, intracerebral e intraventricular. Desenvolvem-se dias, semanas ou meses após o procedimento cirúrgico ${ }^{7,36}$. Têm sido relatados casos de hemorragia intratumoral após esse procedimento cirúrgico $^{38-40,43}$ :

O hematoma extradural é uma complicação rara de derivação ventriculoperitoneal (DVP), sendo considerado menos comum que o hematoma subdu$\mathrm{ral}^{7,11,12,23,25,32}$. Ocorre freqüentemente em pacientes jovens e localiza-se, geralmente, nas regiões frontal e parietal ${ }^{9,32}$. O mecanismo de desenvolvimento do hematoma extradural, nesses casos, seria a rápida redução da pressão intracraniana como conseqüência da superdrenagem de líquido cefalorraquidiano (LCR), resultando na separação da dura-máter do crânio e ruptura de pequenos vasos durais ${ }^{7-11,23,25,32,36,41}$. Outra explicação seria a presença de uma cicatriz na aracnóide - em decorrência de trauma ou infecção prévia - que resultaria em obliteração anatômica de parte do espaço subaracnóideo, causando aderência do cérebro à dura-máter, que pode facilitar a formação do hematoma extradural'. Esses pacientes podem se apresentar assintomáticos, ou com sintomas de mau funcionamento da derivação, ou mesmo de lesão expansiva ${ }^{8,17,23}$. O hematoma extradural deve ser drenado por craniotomia osteoplástica; raramente é necessário trocar o sistema de derivação por um sistema de alta pressão, ou efetuar seu fechamento provisório?

O hematoma subdural, como complicação pósderivação para tratamento de hidrocefalia, tem sido bastante relatado na literatura ${ }^{6}$. Sua incidência é maior que a dos hematomas de outras localizações intracranianas, variando entre $4,5 \%$ e $21 \%$ nas diversas séries $^{2,4,8,14,21,24,28,35}$. Segundo Pudenz e Foltz ${ }^{27}$, a formação do hematoma ocorre dentro de 12 a 24 meses após o procedimento cirúrgico. A hipotensão intracraniana tem sido o fator predisponente mais freqüente na formação do hematoma subdura ${ }^{19}$. Quando um considerável volume de LCR é agudamente drenado do ventrículo, há estiramento das veias-ponte que podem se romper espontaneamente ou após deslocamentos do encéfalo, provocado por trauma craniano de intensidade variável, resultando em formação de hematoma subdural ${ }^{26,6,14,19,22,23,26}$. Hematomas subdurais são freqüentemente relatados em associação a válvulas de baixa pressão; também, têm sido observados em sistemas de pressão mais elevada ${ }^{42} \mathrm{e}$, principalmente, em pacientes submetidos à derivação para tratamento de hidrocefalia de pressão normal $22,26,28$.

Hematoma subdural ocorre freqüentemente em crianças com idade acima de 18 meses, com acentuada desproporção craniofacial e volumosa ventriculomegalia ${ }^{6,14,24}$. O hematoma subdural pós-DVP é, em geral, assintomático ${ }^{3}$ e pode ocorrer após período variável de tempo da instalação da derivação ${ }^{6}$; em crianças, esse período pode ser mais prolongado em virtude da grande reserva de espaço dentro do crânio $\mathrm{e}$, freqüentemente, sugere mau funcionamento do sistema de drenagem. Deve ser suspeitado sempre que os sintomas se desenvolvam em pacientes com válvulas aparentemente funcionantes ${ }^{14}$.

O tratamento dessas situações apresenta certas peculiaridades. A prevenção dessa complicação consiste na utilização de sistemas de drenagem que impeçam acentuadas variações de pressão. O sistema anti-sifão, desenvolvido com essa finalidade, tem-se mostrado, no entanto, incapaz de eliminar a formação de hematomas subdurais ${ }^{16,21,23,26,42}$. Em casos de crianças com idade superior a 18 meses, com grande desproporção craniofacial e volumosa dilatação ventricular, tem sido sugerido que o uso de válvulas de alta pressão com dispositivo anti-sifão apresenta menor incidência de complicações ${ }^{22}$. Para Portnoy e cols. ${ }^{26}$, a aplicação de um sistema anti-sifão com prevenção de pressão intraventricular negativa não diminui a incidência de hematoma subdural. A troca de válvula por outra de pressão maior é indicada em casos de recidiva do hematoma ou em situações especiais ${ }^{6,13,14}$. Dietrich e cols. ${ }^{6}$ trataram um caso com drenagem do hematoma e uso de válvula de pressão regulável, com resultado 
excelente. Em casos de pacientes shunt-dependentes, Portnoy e cols. ${ }^{26}$ idealizaram o sistema de drenagem ventricular e subdural combinadas, sendo a porção ventricular dotada de dispositivo de oclusão temporária e válvula anti-sifão. Em casos de insucesso após todas as alternativas, tem sido indicada a craniotomia seguida de membranectomia da cápsula do hematoma.

Hemorragia intracerebral como complicação de DVP é, também, considerada rara ${ }^{5,20,30,33,37}$. Sua incidência varia de $0,4 \%$ a $4 \%{ }^{29,37}$. Pode ocorrer quando há múltiplas tentativas de punção do ventrículo, colocação imprópria do cateter dentro do parênquima cerebral e perfuração de vasos intracerebrais ${ }^{29}$. Nesses casos, a hemorragia ocorre após a inserção do cateter. Snow e cols..$^{34}$ relataram um caso de hemorragia intracerebral em paciente adulto que foi submetido à DVP para tratamento de hidrocefalia idiopática. A hemorragia ocorreu uma semana após a cirurgia. Esses autores supuseram que o cateter teria provocado erosão na parede de um vaso cerebral. Apesar da baixa incidência, sua mortalidade é elevada, variando de $50 \%$ a $100 \%{ }^{15}$.

Hemorragia intraventricular ocorre raramente como complicação de DVP ${ }^{14,18,30}$. Geralmente acontece quando da inserção do cateter ou da sua retirada, em decorrência de sangramento, por lesão, da artéria ou veia do plexo coróide ou de veias ependimárias. Tem sido mais comum em crianças portadoras de hidrocefalia associada à malformação da veia de Galeno; nestes casos, portanto, a inserção do cateter ventricular deve ser feita no corno frontal ${ }^{31}$.

Hemorragia intratumoral tem sido relatada após drenagem ventricular externa ou DVP ${ }^{38-40,43}$. Segundo Snow e cols. ${ }^{34}$, o mecanismo para a formação dessa hemorragia seria a diminuição súbita da pressão intracraniana, antes elevada, e do desequilíbrio da pressão existente entre os compartimentos supra e infratentoriais. Essa alteração de pressão é transmitida dentro do tumor e resultaria em insuficiência vascular, congestão venosa e, finalmente, hemorragia ${ }^{39,43}$.

Em nossa casuística, dez dos vinte pacientes foram submetidos ao tratamento cirúrgico do hematoma: subdural em seis e extradural em quatro. A cirurgia foi indicada por causa do efeito de massa do hematoma. Quatro pacientes evoluíram para óbito: dois casos em virtude de infecção associada, um por hemorragia intraventricular maciça e outro por complicações sistêmicas.

Para minimizar as complicações hemorrágicas da derivação ventricular, recomendam-se algumas medidas: evitar, ao máximo, a perda de LCR no momento da inserção do cateter ventricular; meticulosa técnica cirúrgica; uso de válvulas de média ou alta pressão; retorno lento da posição deitada para posição sentada e controle pós-operatório realizado por acompanhamento clínico e de imagens ${ }^{3,23}$.

\section{Referências}

1. AGUIAR PH, SHU EBS, FREITAS ABR, LEME RJA, MIURA FK, MARINO Jr R: Causes and treatment of intracranial haemorrhage complicating shunting for paediatric hydrocephalus. Childs Nerv Syst 16:218-21, 2000.

2. ANDERSON FM: Subdural hematoma: a complication of operation for hydrocephalus. Pediatrics 10:11-8, 1952.

3. BALDINI M, PRINCI L, VIVENZA C: Subdural hamatoma secondary to CSF ventricular shunt. Schweiz Arch Neurol Neurochir Psychiatry 127:5-14, 1980.

4. BECKER DP, NULSEN FE: Control of hydrocephalus by valve-regulated venous shunt: avoidance of complications in prolonged shunt maintenance. J Neurosurg 28:215-26, 1968.

5. DERDEYN CP, DELASHAW JB, BROADDUS WC, JANE JA: Detection of shunt-induced intracerebral hemorrhage by postoperative skull films: A report of two cases. Neurosurgey 22:755-7, 1988.

6. DIETRICH U, LUMENTA C, SPRICK C, MAJEWSKI B: Subdural hematoma in a case of hydrocephalus and macrocrania. Childs Nerv Syst 3:242-3, 1987.

7. DRIESEN W, ELIES W: Epidural and subdural haematomas as a complication of internal drainage of cerebrospinal fluid in hydrocephalus. Acta Neurochir (Wien) 30:85-93, 1974.

8. FAULHAUER K, SCHMITZ P: Overdrainage phenomena in shunt treated hydrocephalus. Acta Neurochir (Wien) 15:89-101, 1978.

9. FRERA C: Supratentorial extradural hematomas secondary to ventricular decompression. Acta Neurochir (Wien) 36:107-9, 1977.

10. GULLIKSEN G, HAASE J: Epidural hematoma following a shunt revision. Acta Neurochir (Wien) 36:107-9, 1977.

11. HAFT $H$, LISS $H$, MOUNT L: Massive epidural haemorrhage as a complication of ventricular drainage. J Neurosurg 17:49-54, 1960.

12. HIGAZI J: Epidural haematoma as a complication of ventricular drainage: report of a case and review of the literature. J Neurosurg 20:527-8, 1963.

13. HUBER ZA: Complications of shunt operations performed in 206 children because of communicating hydrocephalus. Zentralbl Neurochir 42:165-70, 1981.

14. ILLINGWORTH RD: Subdural haematoma after the treatment of chronic hydrocephalus by ventriculocaval shunts. J Neurol Neurosurg Psychiatry 33:95-9, 1970.

15. IVAN LP, CHOO SH, VENTUREYRA ECG: Complications of ventriculoatrial and ventriculoperitoneal shunts in a New Children's Hospital. Can J Surg 23:566-8, 1980.

16. JONES R: Experience with antisiphon devices at the Prince of Wales Children's Hospital Childs Brain 5:555, 1979.

17. KALIA KK, SWIFT DM, PANZ D: Multiple epidura! hematomas following ventriculoperi-toneal shunt. Pediatr Neurosurg 19:78-80, 1993.

18. KUWAMURA K, KOKUNAI T: Intraventricular hematoma secondary to a ventriculoperitoneal shunt. Neurosurgery 10:384-6, 1982.

19. MARKWALDER TM: Chronic subdural hematomas: a review. J Neurosurg 54:637-41,1981.

20. MATSUMURAA, SHINOHARAA, MUNEKATAK, MAKI Y: Delayed intracerebral hemorrhage after ventriculoperitoneal shunt. Surg Neurol 24:503-6, 1985.

21. McCULLOUGH DC, FOX JL: Negative intracranial pressure hydrocephalus in adults with shunts and its relationship to the production of subdural hematoma. J Neurosurg 40:372-5, 1974. 
22. McCULLOUGH DC: Symptomatic progressive ventriculomegaly in hydrocephalic with patient shunts and antisiphon devices. Neurosurgery 19:617-9, 1986.

23. MOUSSA AH, SHARMA SK: Subdural haematoma and the malfunctioning shunt. J Neurol Neurosurg Psychiatry 41:759-61, 1978.

24. O'BRIEN M, JOHNSON M: Management of ventricular shunt complications. Contemp Neurosurg 9:1-7, 1987.

25. PEREIRA CU, PORTO MWS, HOLANDARD, ANDRADE WT: Epidural hematoma after ventriculoperitoneal shunt surgery. Report of two cases. Arq Neuropsiquiatr 56:62932, 1998.

26. PORTNOY HD, SCHULTE RR, FOX JL, CROISSANT PD, TRIPP L: Anti-siphon and reversible occlusion valves for shunting in hydrocephalus and preventing post-shunt subdural hematoma. J Neurosurg 38:729-38, 1973

27. PUDENZ RH, FOLTZ EL: Hydrocephalus: Overdrainage by ventricular shunts: $A$ review and recommendations. Surg Neurol 35:200-12, 1991.

28. SAMUELSON S, LONG DM, CHOU SN: Subdura! hematoma as a complication of shunting procedures for normal pressure hydrocephalus. J Neurosurg 37:548$51,1972$.

29. SAVITZ MH, BOBROFF LM: Low incidence of delayed intracerebral hemorrhage secondary to ventriculoperitoneal shunt insertion J Neurosurg 91:32-4, 1999.

30. SAYERS MP: Shunt complications. Clin Neurosurg 23:393-400, 1976.

31. SCHNEIDER SJ, WISOFF JS, EPSTEIN FJ: Complications of ventriculoperitoneal shunt procedures or hydrocephalus associated with vein of Galen malformations in childhood. Neurosurgery 30:706-8, 1992.

32. SENGUPTA RP, HANKINSON J: Extradural haemorrhage. A hazard of ventricular drainage. J Neurol Neurosurg Psychiatry 35:297-303, 1972.

33 SHURIN S, REKATE H: Disseminated intravascular coagulation as a complication of ventricular catheter placement. J Neurosurg 54:264-7, 1981.

34. SNOW RB, ZIMMERMAN RD, DEVINSKY O. Delayed intracerebral hemorrhage after ventriculoperitoneal shunting. Neurosurgery 19:305-7, 1986.
35. STEINBOK P, THOMPSON GB: Complications of ventriculo-vascular shunts: computer analysis of etiological factors. Surg Neurol 5:31-5, 1976.

36. TJAN TG, AARTS NJM: Bifrontal epidural hematoma after shunt operation and posterior fossa exploration: report of a case with survival. Neuroradiology 19:51-3, 1980.

37. UDVARHELYI GB, WOOD JH, JAMES AE, BARTLET D: Results and complications in 55 shunted patients with normal pressure hydrocephalus. Surg Neurol 3: 271-5, 1975.

38. UMANSKY F, KLUGER Y: Traumatic intratumoral hemorrhage secondary to a ventriculoperitoneal shunt. Child's Nerv Syst 4:310-2, 1988.

39. VAQUERO J, CABEZUDO JM, DeSOLA G, NOMBEL.A $\mathrm{L}$ : Intratumoral hemorrhage in posterior fossa tumors after ventricular drainage: report of two cases. J Neurosurg 54:406-8, 1981.

40. WAGA S, SHIMIZU S, SHIMOSAKA S, TOCHIO H: Intratumoral hemorrhage after a ventriculoperitoneal shunting procedure. Neurosurgery 9:249-52, 1981.

41. WEISS RM: Massive epidural hematoma complicating ventricular decompression. Report of a case with survival. J Neurosurg 21:235-6, 1964.

42. YAMADA H: A flow regulating device to control differential pressure in CSF shunt systems. J Neurosurg 57:570-2, 1982

43. ZUCCARELLO M, DOLLO C, CAROLLO C: Spontaneous intratumoral hemorrhage after ventriculoperitoneal shunting. Neurosurgery 16:245-6, 1985.

Original recebido em abril de 2004

Aceito para publicação em setembro de 2004

\section{Endereço para correspondência:}

Carlos Umberto Pereira

Av. Augusto Maynard, 245/404

CEP 49015-380 - Aracaju, SE

E-mail:umberto@infonet.com.br 